\title{
INFLUÊNCIA DA ÉPOCA DE APLICAÇÃO DE NEMATICIDAS EM SOQUEIRAS SOBRE AS POPULAÇÕES DE NEMATÓIDES E A PRODUTIVIDADE DA CANA-DE-AÇÚCAR ( $\left.{ }^{1}\right)$
}

\author{
LEILA LUCI DINARDO-MIRANDA $\left(2^{*}\right)$; JOÃO PAULO PIVETTA $\left({ }^{3}\right)$; \\ JULIANO VILELA FRACASSO $\left({ }^{4}\right)$
}

\begin{abstract}
RESUMO
Com a finalidade de definir a melhor época para aplicação de nematicidas em soqueiras, desenvolveram-se seis experimentos, em canaviais colhidos entre julho e novembro, fazendo aplicação de nematicidas aos 5, 20, 40 ou 60 dias depois da colheita. A aplicação de nematicidas contribuiu para incrementos significativos de produtividade em cinco dos seis experimentos instalados. No experimento em que não se observou incremento significativo de produtividade, as populações de nematóides na

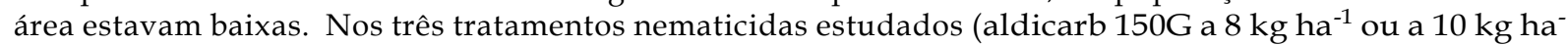
${ }^{1}$ e carbofuran 100G $21 \mathrm{~kg} \mathrm{ha}^{-1}$ ) observou-se desempenho semelhante quanto à redução populacional de nematóides e incrementos de produtividade. Para os experimentos colhidos na época seca, observaram-se os maiores incrementos quando os nematicidas foram aplicados aos 40 ou 60 dias depois do corte. Para o experimento colhido na época chuvosa, os maiores incrementos foram verificados em aplicações feitas aos 20 ou 60 dias depois do corte. Pelos resultados, sugere-se que o período após colheita que se deve aguardar para aplicar o nematicida, a fim de obter os melhores incrementos de produtividade, varia em função da época de colheita do canavial, sendo maior (40 a 60 dias) em canaviais colhidos em época seca e menor (20 dias) em canaviais colhidos na época chuvosa.
\end{abstract}

Palavras-chave: cana-de-açúcar, soqueira, nematóides, controle químico.

\section{ABSTRACT \\ APLLICATION PERIOD EFFECT OF NEMATICIDES IN SUGARCANE RATOON ON NEMATODES POPULATIONS AND SUGARCANE YIELD}

The objective of this work was to define the best period of nematicides application in sugarcane ratoon, Six experiments were carried out in the sugarcane field harvested from July through November during which nematicides were applied at 5, 20, 40 or 60 days after harvesting. Nematicides contributed to increase yield in five of the six experiments. In the experiment in which yield increase was not observed, nematode population was low. The three nematicide treatments (aldicarb 150G at doses 8 kg.ha ${ }^{-1}$ or $_{10}$ kg.ha ${ }^{-1}$ and carbofuran 100G $21 \mathrm{~kg} \cdot \mathrm{ha}^{-1}$ ) promoted similar reductions in the nematode populations and yield increases. In the experiments harvested during the dry season, higher productions were obtained when nematicides were applied at 40 or 60 days after the harvest and, in the experiment harvest at raining season, the highest production was obtained when the nematicides were applied at 20 or 60 days after harvesting. These results suggest that the period following harvest that must be waited before applying nematicidas in sugarcane rations, in order to obtain the best yield increase, varies with the sugarcane harvest time; being best from 40 to 60 days after harvesting in the dry season and 20 days at raining season.

Key words: sugarcane, ratoon, nematodes, chemical control.

$\left({ }^{1}\right)$ Recebido para publicação em 17 de fevereiro de 2006 e aceito em 26 de junho de 2007.

$\left({ }^{2}\right)$ Centro de Cana-de-açúcar (IAC), Caixa postal 206, 14001-970 Ribeirão Preto (SP). E-mail: leiladinardo@iac.sp.gov.br (*) Autora correspondente.

$\left({ }^{3}\right)$ Bayer CropScience Ltda., Av. Presidente Vargas 2001, 14020-260 Ribeirão Preto (SP).

$\left({ }^{4}\right)$ Centro de Cana-de-Açúcar, Fundag/IAC, 14001-970 Ribeirão Preto (SP). 


\section{INTRODUÇÃO}

O uso de nematicidas químicos no plantio de cana-de-açúcar, em áreas infestadas por nematóides, tem sido comum nos últimos anos, devido aos incrementos de produtividade, que podem chegar a cerca de $40 \mathrm{t} \mathrm{ha}^{-1}$, como verificado em diversos dados experimentais (GARCIA al., 1997; DinARDO-MirANDA et al., 1998).

Em soqueiras, o aumento de produtividade decorrente do controle químico de nematóides é menor do que o observado em cana-planta. A menor resposta da cana-soca ao controle de nematóides foi verificada ainda na década de 1980, quando Novaretti et al. (1980) trabalharam com a variedade CB41-76, em área infestada por Meloidogyne javanica (Treub) Chitwood e Pratylenchus zeae Graham. Também VIeIRA et al. (1988), fazendo aplicações de nematicidas na canaplanta e na soqueira subseqüente, não detectaram diferenças de produtividade na soca, entre parcelas tratadas e não tratadas com nematicida, embora houvessem observado diferenças na cana planta de diversas variedades. Em trabalhos mais recentes, envolvendo variedades atualmente em cultivo, DiNARDO-MirANDA et al. (2000) verificaram que os nematicidas carbofuran e terbufós reduziram significativamente as populações de $M$. incognita (Kofoid \& White) Chitwood e de P. zeae, em soca de segundo corte da variedade RB825336, mas o incremento máximo de produtividade, em relação às parcelas testemunhas, foi de apenas 10,2 $\mathrm{t} \mathrm{ha}^{-1}$.

Em uma série de experimentos, nos quais diversos nematicidas foram utilizados, DINARDOMiRANDA et al. (2001) concluíram que, embora os nematicidas tenham reduzido as populações de nematóides nas raízes, não contribuíram de modo consistente para aumentos da produtividade agrícola., Dinardo-Miranda e Garcia (2002) aplicaram nematicidas aos 20, 40 ou 60 dias depois do corte de um canavial da variedade RB835113, infestado por $P$. zeae e verificaram que os nematicidas contribuíram para incrementos de produtividade somente quando aplicados aos $40 \mathrm{ou}$ 60 dias, induzindo os autores a concluir que a época de aplicação de nematicidas, após o corte do canavial, seria fator importante na resposta ao controle químico.

Como há soqueiras colhidas em diferentes épocas ao longo da safra, é necessário estabelecer a melhor época de aplicação de nematicidas para que o controle obtido seja economicamente viável. Dessa forma, o objetivo do presente trabalho foi avaliar a eficácia de nematicidas, aplicados em diferentes épocas após o corte de canaviais colhidos de julho a novembro.

\section{MATERIAL E MÉTODOS}

Foram implantados seis experimentos, utilizando o delineamento em blocos casualizados, com seis repetições e parcelas representadas por seis sulcos de $8 \mathrm{~m}$ de comprimento, espaçados entre si por 1,4 m (ensaios 1, 3, 4 e 5) ou 1,5 m (ensaios 2 e 6). Os tratamentos avaliados foram aldicarb 150G 8 $\mathrm{kg} \mathrm{ha}^{-1}$, aldicarb 150G $10 \mathrm{~kg} \mathrm{ha}^{-1} \mathrm{e}$ carbofuran 100G $21 \mathrm{~kg} \mathrm{ha}^{-1}$, aplicados cerca de 5, 20, 40 e 60 dias depois do corte, e testemunha (sem nematicida). No ensaio 1, os tratamentos nematicidas não foram aplicados cinco dias depois do corte. Desse modo, os tratamentos foram analisados em fatorial $3 \times 4$ (três tratamentos nematicidas em quatro épocas de aplicação após o corte) com uma testemunha adicional, em todos os ensaios. No ensaio 1 , os tratamentos foram analisados em fatorial $3 \times 3$ (três tratamentos nematicidas em três épocas de aplicação) com uma testemunha adicional.

Em todos os ensaios, os nematicidas foram aplicados manualmente, sobre a linha de cana e cobertos com enxada.

As populações de nematóides ocorrentes em cada experimento foram estimadas em três ocasiões: na instalação do experimentos, cerca de 30 dias depois da última aplicação de nematicidas, portanto, aproximadamente aos 90 dias depois do corte, e no período chuvoso (entre janeiro e abril). Para tanto, raízes de plantas de cada parcela foram coletadas e os nematóides, extraídos pela combinação dos métodos de peneiramento com flotação centrífuga em solução de sacarose, de acordo com Coolen e D'Herde (1972). Por ocasião da colheita, feita aproximadamente 12 meses depois do corte anterior, foram obtidas as produtividades agrícolas.

As datas de aplicação dos tratamentos em cada ensaio, das amostragens nematológicas, colheita e outras características importantes estão inseridas na tabela 1.

Para análise estatística, os dados populacionais de nematóides foram transformados em $\log (x+1)$.

A análise estatística dos dados referentes à primeira amostragem foi feita desconsiderando o esquema fatorial dos tratamentos, uma vez que eles ainda não haviam sido aplicados. Para os demais parâmetros (populações de nematóides na segunda e na terceira amostragens e produtividade), as análises foram efetuadas considerando, inicialmente, o esquema fatorial com uma testemunha adicional. Posteriormente, procedeu-se a análise, retirando o tratamento-testemunha.

Todas as médias foram comparadas pelo teste de Tukey. 
Tabela 1. Características gerais dos experimentos

\begin{tabular}{|c|c|c|c|c|c|c|}
\hline Característica & Ensaio 1 & Ensaio 2 & Ensaio 3 & Ensaio 4 & Ensaio 5 & Ensaio 6 \\
\hline Local & Piracicaba & Itirapina & Itirapina & Itirapina & Iracemápo-lis & Santa Rita do Passa Quatro \\
\hline Variedade & RB72454 & SP80-1842 & SP81-3250 & SP80-3280 & RB855536 & RB72454 \\
\hline $\begin{array}{l}\text { Estágio } \\
\left.\text { (n. }{ }^{\circ} \text { de cortes }\right)\end{array}$ & 1 & 3 & 2 & 1 & 1 & 2 \\
\hline $\begin{array}{l}\text { Data do último corte } \\
\text { Aplicações: }\end{array}$ & $25 / 7 / 03$ & $4 / 8 / 04$ & $19 / 8 / 03$ & $8 / 9 / 03$ & $25 / 10 / 03$ & $6 / 11 / 03$ \\
\hline $\begin{array}{l}\text { Época } 1 \text { - data } \\
\text { (dias após o corte) }\end{array}$ & Não tem & $\begin{array}{c}10 / 08 / 04 \\
(6)\end{array}$ & $\begin{array}{c}26 / 08 / 03 \\
(7)\end{array}$ & $\begin{array}{c}11 / 09 / 03 \\
(3)\end{array}$ & $\begin{array}{l}30 / 10 / 03 \\
\quad(5)\end{array}$ & $\begin{array}{l}12 / 11 / 03 \\
(6)\end{array}$ \\
\hline $\begin{array}{l}\text { Época } 2 \text { - data } \\
\text { (dias após o corte) }\end{array}$ & $11 / 8 / 03$ & $\begin{array}{l}30 / 8 / 04 \\
\quad(17)\end{array}$ & $\begin{array}{l}8 / 9 / 03 \\
(26)\end{array}$ & $\begin{array}{l}29 / 9 / 03 \\
(20)\end{array}$ & $\begin{array}{c}19 / 11 / 03 \\
(21)\end{array}$ & $\begin{array}{c}26 / 11 / 03 \\
(25)\end{array}$ \\
\hline $\begin{array}{l}\text { Época } 3 \text { - data } \\
\text { (dias após o corte) }\end{array}$ & $5 / 9 / 03$ & $\begin{array}{l}16 / 9 / 04 \\
(40)\end{array}$ & $\begin{array}{l}29 / 9 / 03 \\
\quad(42)\end{array}$ & $\begin{array}{l}18 / 10 / 03 \\
(41)\end{array}$ & $\begin{array}{l}9 / 12 / 03 \\
\quad(40)\end{array}$ & $\begin{array}{c}11 / 12 / 03 \\
(45)\end{array}$ \\
\hline $\begin{array}{l}\text { Época } 4 \text { - data } \\
\text { (dias após o corte) }\end{array}$ & $25 / 9 / 03$ & $\begin{array}{l}30 / 9 / 04 \\
\quad(60)\end{array}$ & $\begin{array}{c}18 / 10 / 03 \\
(57)\end{array}$ & $\begin{array}{l}5 / 11 / 03 \\
(60)\end{array}$ & $\begin{array}{c}29 / 12 / 03 \\
(57)\end{array}$ & $\begin{array}{c}6 / 1 / 04 \\
(65)\end{array}$ \\
\hline $\begin{array}{l}\text { Amostragens: } \\
\text { 1. }{ }^{a} \text { ou prévia }\end{array}$ & $11 / 8 / 03$ & $10 / 8 / 04$ & $26 / 8 / 03$ & $11 / 9 / 03$ & $30 / 10 / 03$ & $12 / 11 / 03$ \\
\hline $2 .^{\mathrm{a}}$ & $20 / 10 / 03$ & $10 / 11 / 04$ & $18 / 11 / 03$ & $6 / 12 / 03$ & $6 / 2 / 04$ & $20 / 2 / 04$ \\
\hline $3 .^{a}$ & $4 / 2 / 04$ & $14 / 12 / 04$ & $8 / 1 / 04$ & $8 / 1 / 04$ & $16 / 3 / 04$ & $1 .^{\circ} / 4 / 04$ \\
\hline Colheita & $26 / 7 / 04$ & $28 / 7 / 05$ & $17 / 8 / 04$ & $21 / 9 / 04$ & $8 / 10 / 04$ & $21 / 10 / 04$ \\
\hline
\end{tabular}

\section{RESULTADOS E DISCUSSÃO}

Das espécies de nematóides economicamente importantes para a cana-de-açúcar, as detectadas na área do ensaio 1 foram $M$. javanica e $P$. zeae; no ensaio $2, P$. zeae e P. brachyurus; no ensaio 3, M. javanica, $P$. zeae e $P$. brachyurus; no ensaio 4 , $P$. zeae e $P$. brachyurus; e nos ensaios 5 e $6, P$. zeae.

$\mathrm{Na}$ primeira amostragem, realizada por ocasião da implantação dos ensaios, não se detectaram diferenças significativas entre os tratamentos quanto às populações de nematóides e, somente no ensaio 4 , observaram-se diferenças entre os blocos (Tabelas 2 a 7).

Os nematicidas foram eficientes na redução populacional de nematóides em quatro dos seis experimentos, cerca de 30 dias depois da última aplicação. Assim, as populações de Pratylenchus nas parcelas tratadas (médias dos tratamentos nematicidas) estavam significativamente menores que no tratamento testemunha nos ensaios 2 (testemunha $=8250$ exemplares; tratado $=3241$ exemplares $) ; 3$ $($ testemunha $=5006$ exemplares; tratado $=2328$ exemplares); 4 (testemunha $=37280$ exemplares; tratado $=24091$ exemplares) e 5 (testemunha $=6917$ exemplares; tratado $=3162$ exemplares). Entretanto, não se observaram diferenças entre os tratamentos com nematicida e o tratamento testemunha quanto às populações de $M$. javanica e $P$. zeae no ensaio 1 , de $M$. javanica no ensaio 3 e de $P$. zeae no ensaio 6 (Tabelas 2 a 7$)$.

$\mathrm{Na}$ terceira amostragem populacional, efetuada em pleno período chuvoso, quando as populações geralmente atingiram os valores mais elevados, verificou-se que os nematicidas estavam perdendo a eficiência; na maioria dos experimentos não se observaram diferenças entre as parcelas tratadas e a testemunha quanto às populações de nematóides, exceto no ensaio 1 , onde se verificou no tratamento-testemunha significativamente mais exemplares de $M$. javanica que a média dos tratamentos nematicidas (testemunha $=800$ exemplares; tratado $=287$ exemplares) e no ensaio 4 , em relação à Pratylenchus (testemunha $=34767$ exemplares; tratado $=16226$ exemplares).

As análises estatísticas realizadas desconsiderando a testemunha revelaram que os tratamentos nematicidas tiveram desempenho semelhante na redução populacional dos nematóides. Diferenças entre eles foram observadas somente no ensaio 2, nas duas amostragens feitas depois das aplicações e no ensaio 4, na amostragem aos 30 dias depois da última aplicação. No ensaio 2, em parcelas tratadas com aldibarb a $10 \mathrm{~kg} \mathrm{ha}^{-1}$ ocorreram menores populações de $P$. zeae e $P$. brachyurus do que aquelas que receberam carbofuran, enquanto no ensaio 4 , no tratamento com aldicarb a $8 \mathrm{~kg} \mathrm{ha}^{-1}$ observaram-se menores populações dessas mesmas espécies que nas parcelas tratadas com carbofuran. 
Tabela 2. Populações de juvenis de segundo estágio de Meloidogyne javanica (Mj) e de Pratylenchus zeae (Pz) nas raízes $(50 \mathrm{~g})$ da variedade RB72454, em função dos tratamentos e datas de amostragem. Ensaio 1

\begin{tabular}{|c|c|c|c|c|c|c|c|}
\hline \multirow{3}{*}{$\begin{array}{l}\text { Tratamento } \\
\text { nematicida }\end{array}$} & \multirow{3}{*}{$\begin{array}{c}\text { Época da aplicação } \\
\text { Data (dias depois do corte) }\end{array}$} & \multicolumn{6}{|c|}{ Data da amostragem } \\
\hline & & \multicolumn{2}{|c|}{$11 / 8 / 03$} & \multicolumn{2}{|c|}{$20 / 10 / 03$} & \multicolumn{2}{|c|}{$4 / 2 / 04$} \\
\hline & & $\mathrm{Mj}$ & $\mathrm{Pz}$ & $\mathrm{Mj}$ & $\mathrm{Pz}$ & $\mathrm{Mj}$ & $\mathrm{Pz}$ \\
\hline Testemunha & - & 257 & 128 & 217 & 250 & 800 & 316 \\
\hline A8 & Aplicado em 11/8/03 & 166 & 525 & 67 & 167 & 500 & 466 \\
\hline A10 & $(17 \mathrm{ddc})$ & 108 & 333 & 0 & 167 & 600 & 267 \\
\hline $\mathrm{C} 21$ & & 192 & 433 & 167 & 167 & 183 & 683 \\
\hline A8 & Aplicado em 5/9/03 & 283 & 675 & 0 & 167 & 83 & 150 \\
\hline A10 & $(40 \mathrm{ddc})$ & 200 & 358 & 33 & 483 & 33 & 267 \\
\hline $\mathrm{C} 21$ & & 108 & 683 & 100 & 333 & 300 & 167 \\
\hline A8 & Aplicado em 25/9/03 & 8 & 200 & 16 & 183 & 50 & 150 \\
\hline A10 & $(60 \mathrm{ddc})$ & 450 & 342 & 450 & 283 & 516 & 283 \\
\hline $\mathrm{C} 21$ & & 392 & 225 & 0 & 233 & 316 & 600 \\
\hline \multirow[t]{2}{*}{ Média dos tratamentos } & Testemunha & - & - & 217 & 250 & 800 & 316 \\
\hline & Nematicidas & - & - & 93 & 169 & 287 & 337 \\
\hline \multirow[t]{2}{*}{ Valores de F } & Bloco & $1,11^{\mathrm{NS}}$ & $1,92^{\mathrm{NS}}$ & $1,12^{\mathrm{NS}}$ & $0,84^{\mathrm{NS}}$ & $2,85^{*}$ & $0,53^{\mathrm{NS}}$ \\
\hline & Tratamento & $1,24^{\mathrm{NS}}$ & $1,06^{\mathrm{NS}}$ & $0,84^{\mathrm{NS}}$ & $0,60^{\mathrm{NS}}$ & $6,18^{*}$ & $0,28^{\mathrm{NS}}$ \\
\hline CV (\%) & & 100,0 & 27,5 & 173,2 & 62,1 & 72,1 & 65,6 \\
\hline \multirow[t]{3}{*}{ Média dos nematicidas } & A8 & - & - & 28 & 172 & 211 & 255 \\
\hline & A10 & - & - & 161 & 311 & 383 & 272 \\
\hline & $\mathrm{C} 21$ & - & - & 89 & 24 & 266 & 483 \\
\hline \multirow[t]{3}{*}{ Média das épocas de ap } & $2(17)$ & - & - & 78 & 167 & 428 & 472 \\
\hline & $3(40)$ & - & - & 44 & 328 & 139 & 195 \\
\hline & $4(60)$ & - & - & 155 & 233 & 294 & 344 \\
\hline \multirow[t]{4}{*}{ Valores de F } & Bloco & - & - & $1,21^{\mathrm{NS}}$ & $1,01^{\mathrm{NS}}$ & $3,07^{*}$ & $0,67^{\mathrm{NS}}$ \\
\hline & Nematicida & - & - & $0,59^{\mathrm{NS}}$ & $1,75^{\mathrm{NS}}$ & $0,55^{\mathrm{NS}}$ & $0,26^{\mathrm{NS}}$ \\
\hline & Época & - & - & $0,13^{\mathrm{NS}}$ & $1,10^{\mathrm{NS}}$ & 2,09 NS & $2,22^{\mathrm{NS}}$ \\
\hline & $N^{*} \mathrm{E}$ & - & - & $2,97^{*}$ & $0,46^{\mathrm{NS}}$ & $2,30^{+}$ & $1,98^{\mathrm{NS}}$ \\
\hline CV (\%) & & - & - & 162,6 & 62,8 & 79,6 & 68,8 \\
\hline
\end{tabular}

Significância dos valores de F: ** $=1 \% ; *=5 \% ;+=10 \%$ e NS = não significativo.

A8 = Aldicarb 150G $8 \mathrm{~kg} \mathrm{ha}^{-1}$; A10 = Aldicarb 150G $10 \mathrm{~kg} \mathrm{ha}^{-1}$; C21 = Carbofuran $100 \mathrm{G} 21 \mathrm{~kg} \mathrm{ha}^{-1}$.

Nos ensaios 2, 3, 4 e 5 observou-se influência da época de aplicação dos nematicidas sobre as populações de Pratylenchus, na segunda amostragem. A mesma influência foi observada na terceira amostragem, nos ensaios 2, 4 e 5. Nessas situações, as parcelas que receberam os nematicidas na terceira e/ou quarta época de aplicação tiveram populações menores do que aquelas tratadas na primeira época (Tabelas 2 a 7). Esse fato era esperado para todos os ensaios, uma vez que o período ocorrente entre as últimas aplicações e as amostragens foi menor que aquele entre as primeiras aplicações e as amostragens.
Interação entre produtos e épocas foi observada somente em relação à população de $M$. javanica, na segunda amostragem (30 dias depois da última época de aplicação dos nematicidas), no ensaio 1.

Apesar das populações de nematóides não permitirem distinguir com clareza os tratamentos, as produtividades observadas por ocasião da colheita dos ensaios revelaram dados mais significativos.

$\mathrm{Na}$ média, os tratamentos nematicidas propiciaram incrementos significativos de produtividade em todos os ensaios, exceto no ensaio 
5 (Tabela 8). Os incrementos médios (média de todos os tratamentos nematicidas) variaram de $2,1 \mathrm{t} \mathrm{ha}^{-1}$ (ensaio 2) a 8,2 t ha- (ensaio 6). Considerando cada tratamento isoladamente, observaram-se aumentos maiores. Assim, no ensaio 1, utilizando os tratamentos aldicarb $10 \mathrm{~kg} \mathrm{ha}^{-1}$, aplicado 40 ou 60 dias depois do corte e aldicarb $8 \mathrm{~kg} \mathrm{ha}^{-1}$, aplicado aos 60 dias depois do corte, observaram-se incrementos em relação à testemunha de até $11 \mathrm{tha}^{-1}(18,4 \%)$; no ensaio 3 , as duas doses de aldicarb, aplicadas aos 60 dias depois do corte, contribuíram para aumentos até de $18,7 \mathrm{t}$ ha $^{-1}(35,2 \%)$; no ensaio 4, carbofuran aplicado aos 60 dias depois do corte incrementou a produtividade em $13,7 \mathrm{t} \mathrm{ha}^{-1}(20,1 \%)$ e no ensaio 6 , os três nematicidas, aplicados 20 ou 60 dias depois do corte, com incrementos de até $15,6 \mathrm{tha}^{-1}(21,2 \%)$.

Tabela 3. Populações de Pratylenchus zeae e P. brachyurus (Pr) nas raízes (50g) da variedade SP80-1842, em função dos tratamentos e datas de amostragem. Ensaio 2

\begin{tabular}{|c|c|c|c|c|}
\hline \multirow{2}{*}{$\begin{array}{l}\text { Tratamento } \\
\text { nematicida }\end{array}$} & \multirow{2}{*}{$\begin{array}{c}\text { Época da aplicação } \\
\text { Data (dias depois do corte) }\end{array}$} & \multicolumn{3}{|c|}{ Data da amostragem } \\
\hline & & $10 / 8 / 04$ & $10 / 11 / 04$ & $17 / 12 / 04$ \\
\hline Testemunha & & 6650 & 8250 & 6400 \\
\hline A8 & Aplicado em 12/11/03 & 8650 & 2366 & 4566 \\
\hline A10 & $(6 \mathrm{ddc})$ & 6900 & 1816 & 4850 \\
\hline $\mathrm{C} 21$ & & 5533 & 6666 & 3033 \\
\hline A8 & Aplicado em 26/11/03 & 10300 & 5216 & 3650 \\
\hline A10 & $(20 \mathrm{ddc})$ & 4750 & 5816 & 3100 \\
\hline C21 & & 7950 & 4200 & 2333 \\
\hline A8 & Aplicado em 11/12/03 & 6683 & 1883 & 2100 \\
\hline A10 & (35 ddc) & 5400 & 1366 & 616 \\
\hline $\mathrm{C} 21$ & & 6950 & 2983 & 5550 \\
\hline A8 & Aplicado em 6/1/04 & 8616 & 2850 & 1566 \\
\hline A10 & $(60 \mathrm{ddc})$ & 7266 & 1733 & 1233 \\
\hline C21 & & 7966 & 2000 & 2016 \\
\hline \multirow[t]{2}{*}{ Média dos tratamentos } & Testemunha & - & 8250 & 6400 \\
\hline & Nematicidas & - & 3241 & 2168 \\
\hline \multirow[t]{2}{*}{ Valores de F } & Bloco & $1,82^{\mathrm{NS}}$ & $1,79^{\mathrm{NS}}$ & $0,47^{\mathrm{NS}}$ \\
\hline & Tratamento & $0,78^{\mathrm{NS}}$ & $4,34^{*}$ & $2,48^{\mathrm{NS}}$ \\
\hline CV (\%) & & 8,9 & 10,3 & 20,2 \\
\hline \multirow[t]{3}{*}{ Média dos nematicidas } & A8 & - & 3079 & 2071 \\
\hline & A10 & - & 2683 & 2450 \\
\hline & $\mathrm{C} 21$ & - & 3962 & 1983 \\
\hline \multicolumn{5}{|c|}{ Média das épocas de aplicação } \\
\hline & $1(6)$ & - & 3616 & 4150 \\
\hline & $2(20)$ & - & 5077 & 3028 \\
\hline & $3(35)$ & - & 2077 & 2755 \\
\hline & $4(60)$ & - & 2194 & 1605 \\
\hline \multirow[t]{4}{*}{ Valores de F } & Bloco & - & $1,04^{\mathrm{NS}}$ & $0,70^{\mathrm{NS}}$ \\
\hline & Nematicida & - & $3,19 *$ & $3,26^{*}$ \\
\hline & Época & - & $4,06^{*}$ & $3,18^{*}$ \\
\hline & $\mathrm{N}^{*} \mathrm{E}$ & - & $1,41^{* *}$ & $0,84^{\mathrm{NS}}$ \\
\hline CV (\%) & & - & 10,4 & 21,0 \\
\hline
\end{tabular}

Significância dos valores de $\mathrm{F}: * *=1 \% ; *=5 \% ;+=10 \%$ e NS = não significativo.

$\mathrm{A} 8=$ Aldicarb 150G $8 \mathrm{~kg} \mathrm{ha}^{-1}$; A10 = Aldicarb 150G $10 \mathrm{~kg} \mathrm{ha}^{-1}$; C21 = Carbofuran 100G $21 \mathrm{~kg} \mathrm{ha}^{-1}$. 
Tabela 4. Populações de juvenis de segundo estágio de Meloidogyne javanica (Mj) e de Pratylenchus zeae e P. brachyurus

(Pr) nas raízes $(50 \mathrm{~g})$ da variedade SP81-3250, em função dos tratamentos e datas de amostragem. Ensaio 3

\begin{tabular}{|c|c|c|c|c|c|c|c|}
\hline \multirow{3}{*}{$\begin{array}{l}\text { Tratamento } \\
\text { nematicida }\end{array}$} & \multirow{3}{*}{$\begin{array}{c}\text { Época da aplicação } \\
\text { Data (dias depois do corte) }\end{array}$} & \multicolumn{6}{|c|}{ Data da amostragem } \\
\hline & & \multicolumn{2}{|c|}{$26 / 8 / 03$} & \multicolumn{2}{|c|}{$18 / 11 / 03$} & \multicolumn{2}{|c|}{$8 / 1 / 04$} \\
\hline & & $\mathrm{Mj}$ & $\operatorname{Pr}$ & $\mathrm{Mj}$ & $\operatorname{Pr}$ & $\mathrm{Mj}$ & $\operatorname{Pr}$ \\
\hline Testemunha & - & 547 & 2887 & 98 & 5006 & 3617 & 15333 \\
\hline A8 & Aplicado em 26/08/03 & 569 & 1340 & 133 & 1266 & 5733 & 12533 \\
\hline A10 & $(7 \mathrm{ddc})$ & 488 & 557 & 183 & 1933 & 5783 & 11233 \\
\hline $\mathrm{C} 21$ & & 336 & 1934 & 83 & 2466 & 4558 & 21267 \\
\hline A8 & Aplicado em 08/09/03 & 713 & 1651 & 150 & 2500 & 5200 & 7800 \\
\hline A10 & $(20 \mathrm{ddc})$ & 37 & 1659 & 100 & 4333 & 2850 & 12567 \\
\hline $\mathrm{C} 21$ & & 281 & 1350 & 233 & 3683 & 4800 & 8717 \\
\hline A8 & Aplicado em 29/09/03 & 403 & 1499 & 217 & 2166 & 2033 & 13183 \\
\hline A10 & (41 ddc) & 562 & 1923 & 100 & 1466 & 3217 & 17700 \\
\hline $\mathrm{C} 21$ & & 65 & 1657 & 250 & 2300 & 6033 & 13433 \\
\hline A8 & Aplicado em 18/10/03 & 215 & 555 & 250 & 1383 & 2216 & 9050 \\
\hline A10 & $(60 \mathrm{ddc})$ & 164 & 655 & 317 & 2733 & 4566 & 13550 \\
\hline $\mathrm{C} 21$ & & 268 & 1066 & 283 & 1716 & 6217 & 18100 \\
\hline \multirow[t]{2}{*}{ Média dos tratamentos } & Testemunha & - & - & 98 & 5006 & 3617 & 15333 \\
\hline & Nematicidas & - & - & 192 & 2004 & 5033 & 14969 \\
\hline \multirow[t]{2}{*}{ Valores de F } & Bloco & $0,96^{\mathrm{NS}}$ & $0,86^{\mathrm{NS}}$ & $0,23^{\mathrm{NS}}$ & $0,61^{\mathrm{NS}}$ & $1,07^{\mathrm{NS}}$ & $2,26^{+}$ \\
\hline & Tratamento & $1,54^{\mathrm{NS}}$ & 1,50 NS & $0,14^{\mathrm{NS}}$ & $3,50^{+}$ & $0,01^{\mathrm{NS}}$ & $0,90^{\mathrm{NS}}$ \\
\hline $\mathrm{CV}(\%)$ & & 71,0 & 14,6 & 100,1 & 12,7 & 22,7 & 8,8 \\
\hline \multirow[t]{3}{*}{ Média dos nematicidas } & A8 & - & - & 188 & 1829 & 3793 & 10641 \\
\hline & A10 & - & - & 175 & 1641 & 4104 & 13762 \\
\hline & $\mathrm{C} 21$ & - & - & 212 & 2541 & 7202 & 20505 \\
\hline \multicolumn{8}{|c|}{ Média das épocas de aplicação } \\
\hline & $1(7)$ & - & - & 133 & 1888 & 5358 & 15011 \\
\hline & $2(20)$ & - & - & 161 & 3505 & 4283 & 9695 \\
\hline & $3(41)$ & - & - & 189 & 1977 & 3761 & 14772 \\
\hline & $4(60)$ & - & - & 284 & 1944 & 4333 & 13566 \\
\hline \multirow[t]{4}{*}{ Valores de F } & Bloco & - & - & $0,43^{\mathrm{NS}}$ & $0,57^{\mathrm{NS}}$ & $0,91^{\mathrm{NS}}$ & $2,22^{+}$ \\
\hline & Nematicida & - & - & $0,06^{\mathrm{NS}}$ & $1,08^{\mathrm{NS}}$ & $0,52^{\mathrm{NS}}$ & $1,85^{\mathrm{NS}}$ \\
\hline & Época & - & - & $0,98^{\mathrm{NS}}$ & $2,94^{*}$ & $0,16^{\mathrm{NS}}$ & $0,81^{\mathrm{NS}}$ \\
\hline & $\mathrm{N}^{*} \mathrm{E}$ & - & - & $0,37^{\mathrm{NS}}$ & $0,37^{\mathrm{NS}}$ & $0,91^{\mathrm{NS}}$ & $0,90^{\mathrm{NS}}$ \\
\hline $\mathrm{CV}(\%)$ & & - & - & 97,4 & 13,4 & 23,1 & 9,3 \\
\hline
\end{tabular}

Significância dos valores de F: ** $=1 \%{ }^{*}=5 \% ;+=10 \%$ e NS = não significativo.

$\mathrm{A} 8=$ Aldicarb 150G $8 \mathrm{~kg} \mathrm{ha}^{-1}$; A10 = Aldicarb 150G $10 \mathrm{~kg} \mathrm{ha}^{-1}$; C21 = Carbofuran 100G $21 \mathrm{~kg} \mathrm{ha}^{-1}$.

Nota-se que no ensaio 2, a diferença de produtividade entre os tratamentos com nematicidas e a testemunha foi significativa somente a $10 \%$ de probabilidade, enquanto nos demais as diferenças foram significativas a $1 \%$ de probabilidade. A baixa significância da diferença entre a testemunha e os tratamentos nematicidas, no ensaio 2 , e a total falta de resposta ao tratamento químico, no ensaio 5, sugerem que as populações de nematóides nas áreas experimentais não justificavam o uso de nematicidas (Tabela 6). De fato, as populações de P. zeae detectadas na área do ensaio 5 e de $P$. zeae e $P$. brachyurus no ensaio 2, foram, aproximadamente, metade daquelas observadas no ensaio 6 (Tabela 7), no qual a aplicação 
de nematicidas resultou em aumentos significativos de produtividade. Embora as variedades cultivadas nos três ensaios em questão fossem diferentes, todas são suscetíveis a P. zeae (DiNARDo-Miranda et al., 1998), de maneira que a maior resposta ao controle químico no ensaio 6 deve ser atribuída às maiores populações de nematóides na área.

Considerando os ensaios 1, 3, 4 e 6, nota-se uma relação direta entre a grandeza dos incrementos e os níveis populacionais de nematóides. Os maiores incrementos (até 35\%) foram observados no ensaio 3, desenvolvido em área severamente infestada por $M$. javanica e $P$. zeae. Nos ensaios 1, 4 e 6, nos quais as populações foram equivalentes (populações mistas de $M$. javanica e de $P$. zeae, no ensaio 1 ; populações mistas de $P$. zeae e $P$. brachyurus, no ensaio 4 e populações de $P$. zeae, no ensaio 6), os incrementos foram semelhantes, em torno de $20 \%$ (Tabela 8 ).

Tabela 5. Populações de Pratylenchus zeae e de P. brachyurus (Pr) nas raízes (50g) da variedade SP80-3280, em função dos tratamentos e datas de amostragem. Ensaio 4

\begin{tabular}{|c|c|c|c|c|}
\hline \multirow{2}{*}{$\begin{array}{l}\text { Tratamento } \\
\text { nematicida }\end{array}$} & \multirow{2}{*}{$\begin{array}{c}\text { Época da aplicação } \\
\text { Data (dias depois do corte) }\end{array}$} & \multicolumn{3}{|c|}{ Data da amostragem } \\
\hline & & $11 / 9 / 03$ & $6 / 12 / 03$ & $8 / 1 / 04$ \\
\hline Testemunha & - & 5267 & 37280 & 34767 \\
\hline A8 & Aplicado em 11/09/03 & 7150 & 30563 & 20233 \\
\hline A10 & $(3 \mathrm{ddc})$ & 14383 & 29367 & 27033 \\
\hline $\mathrm{C} 21$ & & 6680 & 20350 & 25683 \\
\hline A8 & Aplicado em 29/09/03 & 9117 & 23950 & 18663 \\
\hline A10 & $(21 \mathrm{ddc})$ & 3583 & 20725 & 18583 \\
\hline $\mathrm{C} 21$ & & 7833 & 33354 & 17700 \\
\hline A8 & Aplicado em 18/10/03 & 6117 & 7900 & 11417 \\
\hline A10 & $(40 \mathrm{ddc})$ & 7617 & 20050 & 13100 \\
\hline $\mathrm{C} 21$ & & 4716 & 41350 & 19883 \\
\hline A8 & Aplicado em 05/11/03 & 10433 & 15783 & 15663 \\
\hline A10 & (57 ddc) & 6200 & 22250 & 10583 \\
\hline A21 & & 4183 & 23450 & 23183 \\
\hline \multirow[t]{2}{*}{ Média dos tratamentos } & Testemunha & - & 37280 & 34767 \\
\hline & Nematicidas & - & 24091 & 18477 \\
\hline \multirow[t]{2}{*}{ Valores de F } & Bloco & $2,56^{*}$ & $2,75^{*}$ & $0,55^{\mathrm{NS}}$ \\
\hline & Tratamento & $1,33^{\mathrm{NS}}$ & $6,16^{*}$ & $8,98^{* *}$ \\
\hline $\mathrm{CV}(\%)$ & & 8,0 & 6,8 & 6,1 \\
\hline \multirow[t]{3}{*}{ Média dos nematicidas } & A8 & - & 19549 & 16494 \\
\hline & A10 & - & 23098 & 17325 \\
\hline & $\mathrm{C} 21$ & - & 29626 & 21612 \\
\hline \multicolumn{5}{|c|}{ Média das épocas de aplicação } \\
\hline & $1(3)$ & - & 26760 & 24316 \\
\hline & $2(21)$ & - & 26010 & 18315 \\
\hline & $3(40)$ & - & 23100 & 14800 \\
\hline & $4(57)$ & - & 20494 & 16476 \\
\hline \multirow[t]{4}{*}{ Valores de F } & Bloco & - & $2,46^{*}$ & $0,44^{\mathrm{NS}}$ \\
\hline & Nematicida & - & $3,52^{*}$ & $1,83^{\mathrm{NS}}$ \\
\hline & Época & - & $3,26^{*}$ & $3,67^{*}$ \\
\hline & $\mathrm{N} * \mathrm{E}$ & - & $1,99^{+}$ & $1,07^{\mathrm{NS}}$ \\
\hline $\mathrm{CV}(\%)$ & & - & 7,2 & 6,4 \\
\hline
\end{tabular}

Significância dos valores de F: ** $=1 \% ; *=5 \% ;+=10 \%$ e NS = não significativo.

$\mathrm{A} 8=$ Aldicarb 150G $8 \mathrm{~kg} \mathrm{ha}^{-1} ; \mathrm{A} 10=$ Aldicarb 150G $10 \mathrm{~kg} \mathrm{ha}^{-1} ; \mathrm{C} 21$ = Carbofuran $100 \mathrm{G} 21 \mathrm{~kg} \mathrm{ha}^{-1}$. 
Tabela 6. Populações de Pratylenchus zeae (Pz) nas raízes (50g) da variedade RB855536, em função dos tratamentos e datas de amostragem. Ensaio 5

\begin{tabular}{|c|c|c|c|c|}
\hline \multirow{2}{*}{$\begin{array}{l}\text { Tratamento } \\
\text { nematicida }\end{array}$} & \multirow{2}{*}{$\begin{array}{c}\text { Época da aplicação } \\
\text { Data (dias depois do corte) }\end{array}$} & \multicolumn{3}{|c|}{ Data da amostragem } \\
\hline & & $30 / 10 / 03$ & $6 / 12 / 03$ & $16 / 3 / 04$ \\
\hline Testemunha & - & 3133 & 6917 & 7817 \\
\hline A8 & Aplicado em 30/10/03 & 1700 & 4150 & 6417 \\
\hline A10 & (5 ddc) & 2617 & 4450 & 5650 \\
\hline $\mathrm{C} 21$ & & 2217 & 4517 & 5850 \\
\hline A8 & Aplicado em 19/11/03 & 2600 & 2667 & 2633 \\
\hline A10 & $(25 \mathrm{ddc})$ & 1500 & 1966 & 4350 \\
\hline $\mathrm{C} 21$ & & 1667 & 2016 & 3067 \\
\hline A8 & Aplicado em 9/12/03 & 3017 & 2450 & 4250 \\
\hline A10 & (45 ddc) & 1683 & 2300 & 1867 \\
\hline $\mathrm{C} 21$ & & 1800 & 2133 & 3680 \\
\hline A8 & Aplicado em 29/12/03 & 1167 & 2800 & 2917 \\
\hline A10 & $(65 \mathrm{ddc})$ & 850 & 3283 & 3515 \\
\hline $\mathrm{C} 21$ & & 2267 & 5217 & 7150 \\
\hline \multirow[t]{2}{*}{ Média dos tratamentos } & Testemunha & - & 6917 & 7817 \\
\hline & Nematicidas & - & 3163 & 3279 \\
\hline \multirow[t]{2}{*}{ Valores de F } & Bloco & $1,03^{\mathrm{NS}}$ & $0,53^{\mathrm{NS}}$ & $1,16^{\mathrm{NS}}$ \\
\hline & Tratamento & $1,23^{\mathrm{NS}}$ & $11,18^{* *}$ & 0,29 NS \\
\hline $\mathrm{CV}(\%)$ & & 10,3 & 8,4 & 6,7 \\
\hline \multirow[t]{3}{*}{ Média dos nematicidas } & A8 & - & 3017 & 4054 \\
\hline & A10 & - & 3000 & 3846 \\
\hline & $\mathrm{C} 21$ & - & 3471 & 1937 \\
\hline \multicolumn{5}{|c|}{ Média das épocas de aplicação } \\
\hline & $1(5)$ & - & 4372 & 5972 \\
\hline & $2(25)$ & - & 2216 & 3350 \\
\hline & $3(45)$ & - & 2294 & 3266 \\
\hline & $4(65)$ & - & 3767 & 4527 \\
\hline \multirow[t]{4}{*}{ Valores de F } & Bloco & - & $1,06^{\mathrm{NS}}$ & $0,80^{\mathrm{NS}}$ \\
\hline & Nematicida & - & $0,10^{\mathrm{NS}}$ & $0,01^{\mathrm{NS}}$ \\
\hline & Época & - & $4,27^{* *}$ & $2,83^{*}$ \\
\hline & $\mathrm{N}^{*} \mathrm{E}$ & - & $0,13^{\mathrm{NS}}$ & $1,40^{\mathrm{NS}}$ \\
\hline CV (\%) & & - & 8,6 & 14,9 \\
\hline
\end{tabular}

Significância dos valores de F: ${ }^{* *}=1 \% ; *=5 \% ;+=10 \%$ e NS = não significativo.

A8 = Aldicarb 150G $8 \mathrm{~kg} \mathrm{ha}^{-1}$; A10 = Aldicarb 150G $10 \mathrm{~kg} \mathrm{ha}^{-1}$; C21 = Carbofuran 100G $21 \mathrm{~kg} \mathrm{ha}^{-1}$.

Em que pese as desigualdades entre épocas de colheita e locais de implantação dos ensaios 1 e 6 , a análise dos seus dados revelou a diferença de patogenicidade entre as espécies de nematóides, visto que a mesma variedade foi utilizada (RB72454). Assim, embora as populações de $P$. zeae no ensaio 6 (Tabela 7) sejam numericamente maiores que as verificadas no ensaio 1 para M. javanica (Tabela 2), os aumentos de produtividade decorrentes do uso de
1 todos eles (Tabela 8). Nos ensaios 1, 2, 3 e 4 , desenvolvidos em campos colhidos entre julho e setembro, as maiores produtividades foram observadas quando se aplicaram os nematicidas aos 40 ou 60 dias depois do corte. Os resultados concordam com os anteriormente obtidos por DINARDOMIRANDA e GARCIA (2002), que aplicaram carbofuran e aldicarb, aos 20, 40 ou 60 dias depois do primeiro corte de um canavial da variedade RB835113, infestado por $P$. zeae e colhido em agosto, e observaram que os 
nematicidas contribuíram para incrementos de produtividade, em relação à testemunha, somente quando aplicados aos 40 ou 60 dias após o corte.

As melhores respostas ao controle químico, quando feito aos 40 ou 60 dias depois do corte, em canaviais colhidos na época seca, como observado neste trabalho, devem estar associadas à presença de raízes vivas no período. Como grande parte das raízes da cana-de-açúcar, principalmente as superficiais, responsáveis pela maior parte da absorção, morre na época seca do ano (VAsCONCELOS, 2002), a aplicação de nematicidas entre 40 e 60 dias depois do corte, em canaviais colhidos entre julho e setembro, seria mais adequada pois coincidiria com o período de emissão de novas raízes, principalmente superficiais, propiciando maior absorção dos nematicidas. Além disso, maior quantidade dos produtos estaria disponível para a planta no solo, na primavera/verão, período em que as populações dos nematóides se elevam, favorecidas pelas temperaturas mais altas, chuvas e raízes abundantes.

Tabela 7. Populações de Pratylenchus zeae (Pz) nas raízes (50g) da variedade RB72454, em função dos tratamentos e datas de amostragem. Ensaio 6

\begin{tabular}{|c|c|c|c|c|}
\hline \multirow{2}{*}{$\begin{array}{l}\text { Tratamento } \\
\text { nematicida }\end{array}$} & \multirow{2}{*}{$\begin{array}{c}\text { Época da aplicação } \\
\text { Data (dias depois do corte) }\end{array}$} & \multicolumn{3}{|c|}{ Data da amostragem } \\
\hline & & $12 / 11 / 03$ & $20 / 02 / 02$ & $01 / 04 / 04$ \\
\hline Testemunha & - & 14650 & 12850 & 18317 \\
\hline A8 & Aplicado em 12/11/03 & 10467 & 13080 & 18600 \\
\hline A10 & $(6 \mathrm{ddc})$ & 5850 & 13450 & 17017 \\
\hline C21 & & 10017 & 17480 & 16883 \\
\hline A8 & Aplicado em 26/11/03 & 9833 & 15967 & 16667 \\
\hline A10 & $(20 \mathrm{ddc})$ & 11433 & 16683 & 13850 \\
\hline $\mathrm{C} 21$ & & 8300 & 16850 & 16233 \\
\hline A8 & Aplicado em 11/12/03 & 10683 & 16083 & 13683 \\
\hline A10 & (35 ddc) & 10700 & 17233 & 13333 \\
\hline $\mathrm{C} 21$ & & 7283 & 17417 & 12300 \\
\hline A8 & Aplicado em 06/01/04 & 6483 & 10416 & 17283 \\
\hline A10 & $(60 \mathrm{ddc})$ & 11758 & 13133 & 14367 \\
\hline $\mathrm{C} 21$ & & 8133 & 16250 & 16867 \\
\hline \multirow[t]{2}{*}{ Média dos tratamentos } & Testemunha & - & 12850 & 18317 \\
\hline & Nematicidas & - & 15337 & 15590 \\
\hline \multirow[t]{2}{*}{ Valores de F } & Bloco & $1,08^{\mathrm{NS}}$ & $1,79 \mathrm{NS}$ & $1,16^{\mathrm{NS}}$ \\
\hline & Tratamento & $0,76^{\mathrm{NS}}$ & $4,34^{*}$ & $0,29^{\mathrm{NS}}$ \\
\hline CV $(\%)$ & & 6,9 & 10,3 & 6,7 \\
\hline \multirow[t]{3}{*}{ Média dos nematicidas } & A8 & - & 13887 & 16558 \\
\hline & A10 & - & 15125 & 14642 \\
\hline & $\mathrm{C} 21$ & - & 16999 & 15570 \\
\hline \multicolumn{5}{|c|}{ Média das épocas de aplicação } \\
\hline & $1(6)$ & - & 14670 & 17500 \\
\hline & $2(20)$ & - & 16500 & 10583 \\
\hline & $3(35)$ & - & 16911 & 13105 \\
\hline & $4(60)$ & - & 13266 & 16172 \\
\hline \multirow[t]{4}{*}{ Valores de F } & Bloco & - & $0,86^{\mathrm{NS}}$ & $0,77^{\mathrm{NS}}$ \\
\hline & Nematicida & - & $1,16^{\mathrm{NS}}$ & $1,12^{\mathrm{NS}}$ \\
\hline & Época & - & 2,02 NS & $1,40^{\mathrm{NS}}$ \\
\hline & $\mathrm{N}^{*} \mathrm{E}$ & - & $0,39^{\mathrm{NS}}$ & $0,95^{\mathrm{NS}}$ \\
\hline $\mathrm{CV}(\%)$ & & - & 5,2 & 6,9 \\
\hline
\end{tabular}

Significância dos valores de F: ${ }^{*}=1 \% ;{ }^{*}=5 \% ;+=10 \%$ e NS = não significativo.

A8 = Aldicarb 150G $8 \mathrm{~kg} \mathrm{ha}^{-1}$; A10 = Aldicarb 150G $10 \mathrm{~kg} \mathrm{ha}^{-1}$; C21 = Carbofuran 100G $21 \mathrm{~kg} \mathrm{ha}^{-1}$. 
Tabela 8. Produtividade agrícola (t ha-1) observada nos ensaios, em função dos tratamentos aplicados

\begin{tabular}{|c|c|c|c|c|c|c|c|}
\hline \multirow[b]{2}{*}{ Nematicidas } & \multirow[b]{2}{*}{$\begin{array}{l}\text { Época de aplicação } \\
\text { (dias depois do corte) }\end{array}$} & \multicolumn{6}{|c|}{ Ensaio (data de colheita do canavial) } \\
\hline & & $\begin{array}{c}1 \\
(25 / 7 / 03)\end{array}$ & $\begin{array}{c}2 \\
(4 / 8 / 04)\end{array}$ & $\begin{array}{c}3 \\
(19 / 8 / 03)\end{array}$ & $\begin{array}{c}4 \\
(8 / 9 / 03)\end{array}$ & $\begin{array}{c}5 \\
(25 / 10 / 03)\end{array}$ & $\begin{array}{c}6 \\
(6 / 11 / 03)\end{array}$ \\
\hline Testemunha & & 59,7 & 27,2 & 53,0 & 81,7 & 117,5 & 73,6 \\
\hline A8 & $1(5)$ & - & 29,4 & 53,5 & 82,7 & 121,9 & 79,9 \\
\hline A10 & & - & 29,4 & 60,5 & 86,9 & 119,4 & 79,5 \\
\hline $\mathrm{C} 21$ & & - & 29,7 & 57,7 & 85,4 & 123,1 & 78,2 \\
\hline A8 & $2(20)$ & 69,3 & 25,9 & 53,0 & 88,6 & 122,4 & 87,1 \\
\hline A10 & & 62,9 & 29,1 & 57,2 & 85,6 & 121,0 & 82,7 \\
\hline $\mathrm{C} 21$ & & 65,3 & 27,8 & 58,4 & 91,2 & 123,7 & 82,6 \\
\hline A8 & $3(40)$ & 68,1 & 30,0 & 63,8 & 83,5 & 120,7 & 81,0 \\
\hline A10 & & 70,7 & 28,8 & 62,2 & 95,4 & 122,9 & 75,8 \\
\hline $\mathrm{C} 21$ & & 68,0 & 27,1 & 57,5 & 91,7 & 122,0 & 76,9 \\
\hline A8 & $4(60)$ & 70,6 & 32,3 & 71,7 & 88,2 & 118,1 & 85,2 \\
\hline A10 & & 70,4 & 33,1 & 68,4 & 88,9 & 123,3 & 89,2 \\
\hline $\mathrm{C} 21$ & & 61,4 & 30,0 & 61,1 & 98,1 & 121,2 & 83,6 \\
\hline \multirow[t]{2}{*}{ Média dos tratamentos } & Testemunha & 59,7 & 27,2 & 53,0 & 81,7 & 117,5 & 73,6 \\
\hline & Nematicidas & 67,4 & 29,3 & 60,3 & 88,7 & 121,5 & 81,8 \\
\hline \multirow[t]{2}{*}{ Valores de F } & Bloco & $13,05^{* *}$ & $5,48^{* *}$ & $38,15^{* *}$ & $10,65^{* *}$ & $6,25^{* *}$ & $35,56^{* *}$ \\
\hline & Tratamento & $9,27^{* *}$ & $3,52^{+}$ & $7,83^{* *}$ & $7,41^{* *}$ & $1,77^{\mathrm{NS}}$ & $10,95^{* *}$ \\
\hline $\mathrm{CV}(\%)$ & & 9,1 & 9,3 & 10,4 & 7,1 & 6,0 & 7,2 \\
\hline \multirow[t]{3}{*}{ Média dos nematicidas } & A8 & 69,3 & 29,4 & 60,5 & 85,8 & 120,8 & 83,3 \\
\hline & A10 & 68,0 & 30,1 & 62,1 & 89,2 & 121,7 & 81,8 \\
\hline & $\mathrm{C} 21$ & 64,9 & 28,7 & 58,7 & 91,6 & 122,5 & 80,4 \\
\hline Média das épocas & $1(5)$ & & 29,5 & 57,2 & 85,0 & 121,5 & 79,2 \\
\hline \multirow[t]{3}{*}{ de aplicação } & $2(20)$ & 65,8 & 27,6 & 56,2 & 88,5 & 122,4 & 84,1 \\
\hline & $3(40)$ & 68,9 & 28,6 & 61,1 & 90,2 & 121,9 & 77,9 \\
\hline & $4(60)$ & 67,5 & 31,8 & 67,1 & 91,7 & 120,8 & 86,0 \\
\hline \multirow[t]{4}{*}{ Valores de F } & Bloco & $10,06^{* *}$ & $32,39 * *$ & $3,97^{*}$ & $35,18^{* *}$ & $8,96^{* *}$ & $4,80^{* *}$ \\
\hline & Nematicida & $3,27^{*}$ & $1,79^{\mathrm{NS}}$ & $1,98^{\mathrm{NS}}$ & $5,18^{* *}$ & 0,30 NS & $1,71^{\mathrm{NS}}$ \\
\hline & Época & $2,23^{+}$ & $8,34^{* *}$ & $12,27^{* *}$ & $3,73^{* *}$ & $0,13^{\mathrm{NS}}$ & $8,52^{* *}$ \\
\hline & $N^{*} \mathrm{E}$ & $1,61^{\mathrm{NS}}$ & $1,51^{\mathrm{NS}}$ & $2,76^{*}$ & $2,22^{+}$ & $0,38^{\mathrm{NS}}$ & 0,91 NS \\
\hline $\mathrm{CV}(\%)$ & & 9,0 & 8,9 & 9,8 & 6,8 & 6,1 & 6,9 \\
\hline
\end{tabular}

Significância dos valores de F: ** $=1 \%$; $=5 \% ;+=10 \%$ e NS = não significativo

A8 = Aldicarb 150G $8 \mathrm{~kg} \mathrm{ha}^{-1}$; A10 = Aldicarb 150G $10 \mathrm{~kg} \mathrm{ha}^{-1}$; C21 = Carbofuran 100G $21 \mathrm{~kg} \mathrm{ha}^{-1}$.

Os dados de chuva colhidos no local dos ensaios revelam que, para os ensaios 1, 2, 3 e 4, somente ocorreram chuvas abundantes por ocasião das aplicações feitas aos 60 (ensaios 1 a 3 ) ou aos 40 dias (ensaio 4, Tabela 9). É provável que parte dos produtos aplicados aos 5 ou 20 dias depois do corte, período ainda seco, tenha se decomposto antes da época em que seriam mais úteis, ou seja, durante o período chuvoso.
Em canaviais colhidos na época úmida, as raízes estariam, por ocasião da colheita, em pleno desenvolvimento e, em conseqüência, as populações de nematóides estariam se elevando, justificando a aplicação de nematicidas logo após o corte. Os dados do ensaio 6, no entanto, não esclarecem adequadamente esse parâmetro. No ensaio, localizado em área colhida em novembro, aumentos significativos foram observados em aplicações 
efetuadas aos 20 e aos 60 dias depois do corte e não aos 5 ou 20 dias após, como esperado. Apesar disso, somente nesse ensaio, cuja colheita ocorreu na época chuvosa, observou-se resposta na produtividade quando o nematicida foi aplicado aos 20 dias depois da colheita; esse fato revela a tendência de que o tempo ideal de espera, definido como período após colheita que se deve aguardar para aplicação de nematicidas para obter os melhores incrementos de produtividade, reduz-se gradativamente à medida que a colheita do canavial se aproxima do período chuvoso.

De fato, Silva et al. (2006), trabalhando com a variedade IAC91-5155 em solo infestado por P. zeae, aplicaram nematicidas a dez e 40 dias depois do corte efetuado no início do período chuvoso (20/10/2003); verificaram que os maiores incrementos de produtividade foram obtidos com aplicações feitas a dez dias depois do corte.

A semelhança entre os tratamentos nematicidas, quando foram examinados os dados populacionais, também foi observada ao analisar os dados de produtividade, desconsiderando o tratamento-testemunha. Somente nos ensaios 1 e 4 foram observadas diferenças entre os tratamentos, sendo obtidas no ensaio 1 as maiores produtividades com o tratamento com aldicarb a $8 \mathrm{~kg} \mathrm{ha}^{-1}$, enquanto no ensaio 4 a melhor produtividade foi proporcionada pelo carbofuran (Tabela 8). Como esses dois ensaios foram desenvolvidos em canaviais colhidos em época seca (Tabela 9), não se pode atribuir o melhor comportamento de um produto em determinado ensaio à época de colheita da lavoura. Comportamento semelhante dos dois nematicidas, quando aplicados em soqueiras, já havia sido observado por DINARDOMiranda e Garcia (2002), corroborando os resultados deste trabalho. Esses fatos sugerem que as diferenças entre os produtos quanto à solubilidade (aldicarb 150G tem solubilidade de $9000 \mathrm{ppm}$, enquanto carbofuran 100G, 700 ppm) não é o fator mais importante a ser considerado nos tratamentos em soqueira. A similaridade de resposta aos produtos se deve, provavelmente, ao fato que, quando aplicados em época seca, uma parte muito pequena dos nematicidas deve ser absorvida pela planta logo após a aplicação, uma vez que nesse período ocorre morte de raízes, principalmente as superficiais, responsáveis pela absorção (VAsCONCElos, 2002). Assim, a maior parte dos produtos deve ser absorvida somente na época mais úmida, quando ocorrem novas emissões de raízes; nessas condições, a umidade no solo, possivelmente, é suficiente para disponibilizar às plantas ambos os produtos, até mesmo carbofuran, menos solúvel. Por outro lado, se aplicados na época úmida, os nematicidas provavelmente são rapidamente absorvido pela planta, uma vez que há raízes superficiais novas para a pronta absorção, minimizando eventuais perdas por lixiviação, inclusive do produto mais solúvel (aldicarb). Portanto, como o aproveitamento dos nematicidas está diretamente ligado à capacidade da planta em absorvêlos, e esta, à emissão de raízes superficiais novas, a solubilidade dos nematicidas, que permitiria maior disponibilidade de aldicarb em época seca ou favoreceria carbofuran em condições mais úmidas, parece ter menor importância.

Reforça as afirmações acima o fato de que não se observaram, com freqüência, interações significativas entre o tratamento nematicida e a época de aplicação. No ensaio 3, o tratamento com carbofuran resultou em produtividades semelhantes, em quaisquer das épocas de aplicação, enquanto os tratamentos com aldicarb, a 8 ou a $10 \mathrm{~kg} \mathrm{ha}^{-1}$, resultaram em maiores produtividades quando aplicados aos 60 dias depois do corte. No ensaio 4, as diferenças foram significativas somente a $10 \%$ de probabilidade. Nesse caso, carbofuran e aldicarb a 10 $\mathrm{kg} \mathrm{ha}^{-1}$ propiciaram maiores produtividades quando aplicados aos 60 dias depois do corte, enquanto com aldicarb a $8 \mathrm{~kg} \mathrm{ha}^{-1}$ a produtividade foi semelhante nas quatro épocas de aplicação (Tabela 8).

Tabela 9. Volume de chuvas ( $\mathrm{mm}$ ) nos diferentes períodos de condução de cada experimento

\begin{tabular}{|c|c|c|c|c|c|c|}
\hline \multirow{2}{*}{ Período } & \multicolumn{6}{|c|}{ Ensaio } \\
\hline & 1 & 2 & 3 & 4 & 5 & 6 \\
\hline Nos 30 dias anteriores ao corte & 18 & 2 & 6 & 5 & 90 & 176 \\
\hline Do corte à primeira aplicação ( \pm 5 dias depois do corte $)$ & 0 & 6 & 5 & 1 & 7 & 17 \\
\hline Do corte à segunda aplicação ( \pm 20 dias depois do corte $)$ & 13 & 11 & 5 & 6 & 139 & 43 \\
\hline Do corte à terceira aplicação ( \pm 40 dias depois do corte) & 16 & 13 & 11 & 67 & 319 & 266 \\
\hline Do corte à quarta aplicação ( \pm 60 dias depois do corte $)$ & 24 & 17 & 77 & 100 & 387 & 449 \\
\hline
\end{tabular}




\section{CONCLUSÕES}

1. O tratamento nematicida contribuiu para aumentos significativos de produtividade em cinco dos seis ensaios realizados.

2. No ensaio onde não se observou incremento significativo de produtividade devido à aplicação de nematicidas, as populações de nematóides estavam baixas;

3. Os três tratamentos nematicidas estudados (aldicarb 150G a $8 \mathrm{~kg} \mathrm{ha}^{-1}$ ou a $10 \mathrm{~kg} \mathrm{ha}^{-1}$ e carbofuran 100G $21 \mathrm{~kg} \mathrm{ha}^{-1}$ ) tiveram desempenho semelhante quanto à redução populacional de nematóides e incrementos de produtividade;

4. Para os experimentos colhidos na época seca, os maiores aumentos foram observados quando o nematicida foi aplicado aos 40 ou 60 dias depois do corte; para o ensaio colhido na época chuvosa, obtiveram-se maiores incrementos em aplicações feitas aos 20 ou 60 dias depois do corte, sugerindo o período após colheita que se deve aguardar para aplicações de nematicidas para obter os melhores incrementos de produtividade, varia em função da época de colheita do canavial.

\section{REFERÊNCIAS}

COOLEN, W.A.; D'HERDE, C.J. A method for the quantitative extraction of nematodes from plant tissue. Ghent: State Nematology and Entomology Research Station,1972. 77p.

DINARDO-MIRANDA, L.L.; GARCIA, V. Efeito da época de aplicação de nematicidas em soqueira de cana-de-açúcar. Nematologia Brasileira, Brasília, v. 26, n. 2, p. 177-180, 2002.
DINARDO-MIRANDA, L.L.; GARCIA, V.; MENEGATTI, C.C. Controle químico de nematóides em soqueiras de cana-deaçúcar. Nematologia Brasileira, Brasília, v.24, n.1, p.55-58, 2000.

DINARDO-MIRANDA, L.L.; MENEGATTI, C.C.; GARCIA, V.; PIVETTA, J.P. Eficiência de nematicidas em soqueiras de canade-açúcar. STAB - Açúcar, Álcool e Subprodutos, Piracicaba, v.19, n.16, p.30-33, 2001.

DINARDO-MIRANDA, L.L.; MENEGATTI, C.C.; GARCIA, V.; SILVA, S.F.; ODORISI, M. Reação de variedades de cana-deaçúcar a Pratylenchus zeae. STAB - Açúcar, Álcool e Subprodutos, Piracicaba, v.17, n.2, p.39-41, 1998.

GARCIA, V.; SILVA, S.F.; DINARDO-MIRANDA, L.L. Comportamento de variedades de cana-de-açúcar em relação a Meloidogyne incognita. Revista Nacional do Álcool e Açúcar, São Paulo, v.17, n.87, p.14-19, 1997.

NOVARETTI, W.R.T.; LORDELLO, L.G.E.; NELLI, E.J.; CARDERAN, J.O. Viabilidade econômica do nematicida carbofuran na cultura da cana-de-açúcar - cana de segundo corte. In: REUNIÃO BRASILEIRA DE NEMATOLOGIA, 4., 1980, Piracicaba. Piracicaba: Sociedade Brasileira de Nematologia, 1980. p.179-196. (Publicação n. ${ }^{\circ}$ 4).

SILVA, M.A.; PINCELLI, R.P.; DINARDO-MIRANDA. L.L. Efeito da aplicação de nematicida na soqueira de cana-deaçúcar, em diferentes épocas, sobre a população de Pratylenchus zeae e atributos biométricos e tecnológicos da cultura. Nematologia Brasileira, Brasília, v.30, n.1, p.29-34, 2006.

VASCONCELOS, A.C. Desenvolvimento do sistema radicular e da parte aérea de socas de cana-de-açúcar sob dois sistemas de colheita: crua mecanizada e queimada manual. 2002. 141p. Tese (Doutorado) - FCAV/UNESP, Jaboticabal.

VIEIRA, A.S.; AGUILLERA, M.M.; MATSUOKA, S. Reações de genótipos de cana-de-açúcar da sigla RB a nematóides em condições de infestação natural. Nematologia Brasileira, Brasília, v.12, n.1, p.19-20, 1988. 